\title{
Serum Hepcidin: A Direct Link Between Anemia of Inflammation and Coronary Artery Atherosclerosis in Patients with Rheumatoid Arthritis
}

\author{
MANAL ALY ABDEL-KHALEK, AMAL MOHAMAD EL-BARBARY, SALWA ABDEL-MONEIM ESSA, \\ and ABEER SAEED GHOBASHI
}

ABSTRACT. Objective. To investigate the role of hepcidin as an inducer of anemia of inflammation in patients with rheumatoid arthritis (RA), and its correlation to coronary artery atherosclerosis.

Methods. Our study included 60 patients with RA and 20 healthy controls. Anemic RA patients with serum transferrin receptors/log ferritin (sTfR-F) index value $<1.5$ were classified as having pure anemia of chronic disease (ACD), and patients with sTfR-F index value $>1.5$ were classified as having anemia of chronic disease with coexistent iron deficiency anemia (ACD+IDA). Measurements were taken for Disease Activity Score for 28 joints (DAS28), Modified Health Assessment Questionnaire (MHAQ), erythrocyte sedimentation rate (ESR), high sensitivity C-reactive protein (hsCRP), rheumatoid factor (RF), lipid profile, serum interleukin 6 (IL-6), tumor necrosis factor- $\alpha$, iron studies, and serum hepcidin. Coronary calcium score (CCS) was measured using multislice spiral computed tomography as a marker of atherosclerosis.

Results. Serum hepcidin was found to be higher in anemic patients with RA than in controls $(\mathrm{p}<0.001)$, and higher in the pure ACD subgroup than in the ACD+IDA subgroup $(\mathrm{p}<0.001)$. Hepcidin concentration was positively correlated with disease duration, ESR, hsCRP, RF, DAS28, MHAQ, serum ferritin, IL-6, and mean CCS and inversely correlated with hemoglobin, sTfR, and the sTfR-F index.

Conclusion. Hepcidin can be considered a key inducer of anemia of inflammation in patients with RA. This inflammation was proved to be directly linked to coronary artery atherosclerosis. The correlations between serum hepcidin with disease activity and IL-6 raise the possibility of using it as a surrogate marker for disease activity. (First Release Sept 1 2011; J Rheumatol 2011;38:2153-9; doi:10.3899/ jrheum.110339)

\section{Key Indexing Terms: RHEUMATOID ARTHRITIS ATHEROSCLEROSIS}

Rheumatoid arthritis (RA) is a systemic disease that in its more severe form may include rapidly progressive multisystem inflammation. Anemia occurs in $31.5 \%$ of patients with RA, about 3 times the rate in the general population ${ }^{1}$. Although some cases of anemia can be attributed to iron deficiency (ID) secondary to nonsteroidal antiinflammatory drug-related gastrointestinal bleeding, up to $70 \%$ of anemia occurring in patients with RA is attributed to anemia of chronic disease $(\mathrm{ACD})^{2}$.

The leading cause of anemia in RA is chronic inflammation, with the pattern of a normocytic normochromic anemia. It is associated with decreased serum iron and total iron-bind-

From the Department of Rheumatology and Rehabilitation, the Department of Clinical Pathology, and the Department of Radiology, Faculty of Medicine, Tanta University, Tanta, Gharbeia, Egypt.

M.A. Abdel-Khalek, MD, Assistant Professor; A.M. El-Barbary, MD, Lecturer, Department of Rheumatology and Rehabilitation; S.A-M. Essa, MD, Assistant Professor, Department of Clinical Pathology; A.S. Ghobashi, MD, Professor, Department of Radiology, Tanta University. Address correspondence to Dr. A.M. El-Barbary, Tanta Faculty of Medicine, Rheumatology and Rehabilitation Department, Elgesh Street, Tanta, Gharbeia,Egypt.E-mail: ml_barbary@yahoo.com Accepted for publication June 1, 2011.

\section{ANEMIA OF INFLAMMATION} HEPCIDIN

ing capacity (TIBC), but iron store is increased or normal ${ }^{3}$. Inflammatory cytokines contribute to the development of ACD in RA by inhibiting both erythropoietin production and bone marrow erythropoiesis. Interleukin 6 (IL-6) appears to be the central mediator of ACD in RA, through increased generation of hepcidin and the resultant alterations in iron metabolism ${ }^{4}$.

Hepcidin is a liver-derived acute-phase reactant that closely regulates iron homeostasis by controlling the release of iron from cells $s^{5}$. As the key regulator of transmembrane iron transport, hepcidin controls the absorption of iron in the intestine, the mobilization of iron from hepatic stores, and iron recycling by macrophages ${ }^{6}$. It has been suggested that hepcidin is the key mediator of $\mathrm{ACD}$; its production in either serum or urine demonstrates a strong correlation with serum ferritin concentration ${ }^{7}$.

Cardiovascular disease (CVD) is a major contributor to increased mortality among patients with RA. Increased CV risk among patients with RA is not fully explained by traditional CV risk factors alone. Results of many studies have suggested a more direct link between atherogenesis and inflammatory cytokines such as IL- 6 , tumor necrosis factor- $\alpha$ 
(TNF- $\alpha$ ), and IL-1. Evidence suggests a central role for IL-6 in the development of $\mathrm{CVD}^{8}$. Coronary calcification is a marker of atherosclerosis that can be quantified with the use of cardiac computed tomography and is proportional to the extent and severity of atherosclerotic disease ${ }^{9}$.

Our aim was to investigate the role of hepcidin as an inducer of anemia of inflammation in patients with RA, and its correlation with coronary artery atherosclerosis in those patients.

\section{MATERIALS AND METHODS}

Study subjects. Sixty patients with RA were selected from the outpatient clinic of the Rheumatology and Rehabilitation Department, Tanta University Hospitals, Egypt. They fulfilled the 2010 American College of Rheumatology/European League Against Rheumatism classification criteria for RA ${ }^{10}$. All patients were taking disease-modifying antirheumatic drugs (DMARD), low-dose corticosteroids, and/or nonsteroidal antiinflammatory drugs. In addition, 20 healthy volunteers matched in age and sex were included.

We excluded patients with other concomitant hematological diseases such as thalassemia and sickle cell anemia; lung, kidney, or liver diseases (cirrhosis or alcoholic hepatitis and those with alanine aminotransferase or aspartate aminotransferase levels $>2$-fold above normal); acute or chronic infections or malignancy; current pregnancy or delivery within 6 months; participants who received blood transfusion, erythropoietin, or iron treatment; and patients with a recent history of bleeding. Also excluded were patients with RA who had hypertension (systolic blood pressure $>150 \mathrm{~mm} \mathrm{Hg}$ and/or diastolic blood pressure $>90 \mathrm{~mm} \mathrm{Hg}$ ) or conditions that affect the lipid profile such as diabetes mellitus, hypothyroidism, Cushing's syndrome, obesity (body mass index $>30$ ), current smokers, history of familial dyslipidemia, or myocardial infarction during the last 6 months. In addition, we excluded patients receiving medications affecting lipid metabolism such as lipid-lowering drugs, oral prednisolone $>10 \mathrm{mg} /$ day, B-blockers, oral contraceptives, estrogen, progestin, thyroxin, and vitamin $\mathrm{E}$.

Our study was approved by the institutional ethics committee, and all participants gave informed consent.

Clinical assessment. Disease activity in patients with RA was assessed by measuring the 28-joint Disease Activity Score (DAS28). Components of DAS28 are erythrocyte sedimentation rate (ESR), patient-assessed global score $(0-100)$, and swollen and tender joint counts $(0-28)^{11}$. The Modified Health Assessment Questionnaire (MHAQ), a standard 8-question instrument, was used to assess functional capacity based on the difficulty in performing activities of daily living. This questionnaire is scored from 0 to 3 , with higher scores indicating lower functional capacity ${ }^{12}$.

Sampling. A standardized sampling protocol was used for hepcidin analysis that included standardization of time of blood withdrawal, similar to that for the assessment of serum iron levels or transferrin saturation, because of hepcidin's circadian rhythm ${ }^{13}$. After $12 \mathrm{~h}$ of overnight fasting, $7 \mathrm{ml}$ of venous blood were withdrawn from patients and control subjects at 9:00 AM. Of those samples, $1.6 \mathrm{ml}$ blood was transferred into a tube containing $0.4 \mathrm{ml}$ sodium citrate for determination of ESR, $1 \mathrm{ml}$ was placed in EDTA for a complete blood count, and the rest of the blood was delivered in a plain glass tube, allowed to clot at room temperature, centrifuged at $2000 \mathrm{rpm}$ for $10 \mathrm{~min}$, and the serum was separated. Lipid profile, serum iron, TIBC, ferritin, and rheumatoid factor $(\mathrm{RF})$ were determined immediately and aliquots of the rest of the serum were stored at $-70^{\circ} \mathrm{C}$ until the assay of the high sensitivity C-reactive protein (hsCRP), IL-6, TNF- $\alpha$, serum transferrin receptors (sTfR), and serum hepcidin.

The following laboratory data were collected for both patients and controls: complete blood count using Advia 60 cell counter (Siemens Healthcare Diagnostics, Erlangen, Germany); ESR mm/h by Westergren method; quantitative measurement of CRP concentration using highly sensitive ELISA sandwich technique (DiaMed Eurogen, Tessenderlo, Belgium), with the concentration of CRP directly proportional to the optical density; RF, determined by nephelometry (Behring Diagnostics GmbH, Liederbach, Germany); values $\geq$ $15 \mathrm{IU} / \mathrm{ml}$ were considered abnormal, according to manufacturer's recommendations; lipid profile including serum triglycerides (TG), total cholesterol (TC), high-density lipoprotein cholesterol (HDL-C), and low-density lipoprotein cholesterol (LDL-C) concentrations, determined by the corresponding colorimetric methods using kits supplied by bioMérieux Vitek Inc. (Chardonnieres-les-Dains, France); serum apolipoprotein B (apoB) and A-1 were measured by immunonephelometry by kits supplied from Behring; quantitative measurement of serum IL- 6 and TNF- $\alpha$ using ELISA with the quantitative sandwich enzyme immunoassay technique from a commercially available kit and the Quantikines immunoassay supplied by R\&D Systems (Minneapolis, MN, USA), according to the manufacturer's instructions; iron studies, i.e., colorimetric estimation of serum iron and TIBC (BioMerieux, France), transferrin saturation calculated and expressed as percentage of transferrin saturation $(\mathrm{TfS})=$ serum iron/TIBC $\times 100$ ), estimation of serum ferritin using an ELISA kit (DRG International Inc., Mountainside, NJ, USA), serum soluble transferrin receptors (sTfR) level measured by quantitative enzyme immunoassay determination with DiaMed kits, and the ratio of sTfR to log ferritin (sTfR-F index) was calculated; and hepcidin assessment was performed with a hepcidin ELISA (EIA-4705) kit (DRG Diagnostic GmbH, Marburg, Germany) ${ }^{14}$.

The DRG hepcidin ELISA kit is a solid-phase type based on the principle of competitive binding. The microtiter wells are coated with a monoclonal antibody directed toward the antigenic site of bioactive hepcidin 25 molecule. Endogenous hepcidin of a patient sample competes with the added hepcidinbiotin conjugate for binding to the coated antibody. After incubation, the unbound conjugate was washed off, followed by incubation with a streptavidin-peroxidase enzyme complex and a second wash step. Substrate solution was added, resulting in color development that was stopped after a short incubation. The color intensity developed is reversely proportional to the hepcidin concentration in the patient sample.

Determination of anemia subgroups. Anemia in patients with RA was determined as hemoglobin ( $\mathrm{Hb})$ levels $<13.5 \mathrm{mg} / \mathrm{dl}$ for men and $<12 \mathrm{mg} / \mathrm{dl}$ for women ${ }^{15}$. Of the anemic patients with RA, we used the sTfR-F index cutoff levels to determine concomitant iron deficiency anemia. Patients with sTfR-F index value $<1.5$ were classified as having pure anemia of chronic disease (pure ACD) and patients with sTfR-F index value $>1.5$ were classified as having anemia of chronic disease with coexistent iron deficiency anemia (ACD+IDA $)^{16}$.

Coronary calcium score (CCS). CCS was measured using multislice spiral computed tomography with a dedicated cardiac package (GE Healthcare, Amersham, UK). The images were noncontrast electrocardiogram-gated, with each slice triggered at $80 \%$ of the patient's R-R interval, so that the images were obtained at the same point of diastole. The images were taken at 2-2.5 $\mathrm{mm}$ section thickness. Usually 40-60 slices were obtained. Each slice was examined for calcium deposits. The area of calcium and its peak density were determined and added to calculate the selective and total score. The extent of coronary artery calcification was calculated as described by Agatston, et al ${ }^{17}$ by multiplying the area of each calcified lesion by a weighting factor corresponding to the peak pixel attenuation for each lesion to yield a lesion-specific calcium score. The sum of all individual plaque scores represented a patient's total calcium score. Coronary calcium scores were classified into 5 categories $^{18}$ : $0=$ no evidence of plaque; $1-10=$ minimal evidence of plaque; $11-100$, mild evidence of plaque; $101-400$, moderate evidence of plaque, and over 400, extensive evidence of plaque.

Statistical analysis. All data were analyzed using SPSS software (version 11, SPSS Inc., Chicago, IL, USA). Baseline characteristics are presented as mean \pm SD for the continuous variables, and as frequency and percentage for the discrete ones. Comparisons between groups were conducted using the Student $\mathrm{t}$ test. Comparisons between subgroups were conducted using ANOVA. Correlation between variables was examined using Pearson's correlation coefficient. Multiple linear regression analysis was used to determine the independent predictors of serum hepcidin levels. P value $<0.05$ was considered statistically significant. 


\section{RESULTS}

The demographic, clinical, laboratory, and radiographic data for patients with RA and controls are summarized in Table 1. There were 57 women and 3 men; ages ranged from 32 to 52 years (mean $42.15 \pm 6.95$ yrs). Meanwhile, 18 controls were women and 2 were men; ages ranged from 34 to 50 years (mean $43.7 \pm 7.89 \mathrm{yrs}$ ). There were no significant differences in age, sex, and mean body mass index between patients with RA and controls.

At the time of our study, all patients with RA were taking DMARD, with 18 patients (30\%) using methotrexate 20-30 $\mathrm{mg} /$ week as monotherapy, 42 patients $(70 \%)$ receiving a combination of methotrexate therapy with hydroxychloroquine $400 \mathrm{mg} /$ day or sulfsalazine 1.5-2 g/day. Nonsteroidal antiinflammatory drugs were being used by 30 patients $(50 \%)$ and low-dose prednisolone ( $\leq 7.5 \mathrm{mg} /$ day) by 20 patients $(33.3 \%)$.

Patients with RA exhibited mild dyslipidemia characterized by a significantly higher baseline TC, LDL-C, TG, and apoB compared to controls. In addition, HDL-C levels and apolipoprotein A-I (apoA-1) were significantly lower com-

Table 1. Demographic, laboratory, and radiographic measures in patients with rheumatoid arthritis (RA) and controls. Values represent the mean \pm SD unless otherwise noted.

\begin{tabular}{lcc}
\hline Characteristic & RA Patients, $\mathrm{n}=60$ & Controls, $\mathrm{n}=20$ \\
\hline Age, yrs & $42.15 \pm 6.95$ & $43.7 \pm 7.89$ \\
Sex, women/men & $57 / 3$ & $18 / 2$ \\
Body mass index, kg/m ${ }^{2}$ & $25.8 \pm 3.7$ & $25.5 \pm 3.3$ \\
DAS28 & $4.69 \pm 1.14$ & - \\
ESR, mm/h & $39.23 \pm 14.54^{*}$ & $8.10 \pm 3.38$ \\
hsCRP, mg/l & $7.19 \pm 3.55^{*}$ & $1.7 \pm 0.47$ \\
Rheumatoid factor, IU/ml & $83.57 \pm 33.40^{*}$ & $7.5 \pm 2.56$ \\
Total cholesterol, mg/dl & $233.08 \pm 29.85^{*}$ & $177.20 \pm 14.09$ \\
Triglyceride, mg/dl & $132.13 \pm 47.40^{*}$ & $91.30 \pm 19.80$ \\
LDL-C, mg/dl & $137 \pm 25.54^{*}$ & $124 \pm 16.46$ \\
HDL-C, mg/dl & $44.63 \pm 9.19^{*}$ & $54.50 \pm 11.81$ \\
TC/HDL-C & $5.66 \pm 1.04^{*}$ & $3.4 \pm 0.27$ \\
LDL/HDL & $3.40 \pm 0.93^{*}$ & $2.49 \pm 0.81$ \\
ApoB, g/l & $121.82 \pm 9.92^{*}$ & $90 \pm 29$ \\
ApoA-, $\mathrm{g} / \mathrm{l}$ & $125.35 \pm 17.47^{*}$ & $149 \pm 43$ \\
Hemoglobin, g/dl & $9.31 \pm 2.86^{*}$ & $14.1 \pm 0.72$ \\
Serum iron, $\mu \mathrm{g} / \mathrm{l}$ & $58.33 \pm 25.79^{*}$ & $98 \pm 30$ \\
Ferritin, $\mu \mathrm{g} / \mathrm{l}$ & $105.85 \pm 105.76^{*}$ & $73.15 \pm 44.1$ \\
TIBC, $\mu \mathrm{g} / \mathrm{dl}$ & $319.83 \pm 97.85^{*}$ & $233.2 \pm 56.2$ \\
TfS, \% & $18.57 \pm 17.59^{*}$ & $29.15 \pm 20.5$ \\
sTfR, $\mu \mathrm{g} / \mathrm{ml}$ & $3.84 \pm 2.64^{*}$ & $2.17 \pm 0.86$ \\
sTfR-F index & $3.65 \pm 3.66^{*}$ & $1.47 \pm 0.61$ \\
Tumor necrosis factor- $\alpha, \mathrm{pg} / \mathrm{ml}$ & $6.87 \pm 2.92^{*}$ & $3.71 \pm 0.97$ \\
Interleukin $6, \mathrm{pg} / \mathrm{ml}$ & $28.64 \pm 17.82^{*}$ & $5.1 \pm 3.0$ \\
Hepcidin, ng/ml & $426.33 \pm 144.49^{*}$ & $312.5 \pm 144.98$ \\
CCS, Agatston units & $126 \pm 115.23^{*}$ & $4.7 \pm 4.03$ \\
\hline
\end{tabular}

$* \mathrm{p}<0.001$ compared to controls. DAS28: Disease Activity Score for 28 joints; ESR: erythrocyte sedimentation rate; hsCRP: high sensitivity C-reactive protein; LDL-C: low-density lipoprotein cholesterol; HDL-C: high-density lipoprotein cholesterol; ApoB: apolipoprotein B; TIBC: total iron binding capacity; TfS: transferrin saturation; sTfR-F: serum transferrin receptor/log ferritin; CCS: coronary calcium score. pared to controls. As a consequence, the atherogenic ratio of TC/HDL-C as well as that of LDL-C/HDL-C was significantly higher in patients with RA compared to controls.

In patients with RA, laboratory measurements including ESR, hsCRP, TNF- $\alpha$, IL-6, and serum hepcidin levels were significantly higher compared to controls. In addition, mean CCS were significantly higher in patients with RA compared to controls ( $\mathrm{p}<0.001)$.

Regarding iron status in patients with RA, levels of $\mathrm{Hb}$, serum iron, and TfS were significantly lower than controls. Meanwhile, serum ferritin, TIBC, sTfR concentrations, and sTfR-F index were significantly higher than controls.

Out of 60 patients with RA, 40 patients $(66.6 \%)$ had anemia. Of them, $16(40 \%)$ had a pure ACD (sTfR-F index value $<1.5)$, and $24(60 \%)$ had ACD with coexistent IDA (ACD+IDA; sTfR-F index value > 1.5).

Table 2 gives laboratory data of the anemic compared to nonanemic RA subgroups. Anemic RA subgroups showed significantly higher DAS28, ESR, hsCRP, TNF- $\alpha$, and MHAQ levels than the nonanemic group. Meanwhile, the difference between those levels in the ACD+IDA subgroup compared to the pure ACD subgroup was statistically insignificant.

$\mathrm{Hb}$ was significantly decreased in anemic patients. The decrease in $\mathrm{Hb}$ was much more in the ACD+IDA subgroup as compared to the pure ACD subgroup. Serum iron was reduced in both anemia subgroups; however, there was no significant difference between them. TIBC was significantly increased and transferrin saturation was significantly decreased in the ACD+IDA subgroup as compared to the pure ACD subgroup, while serum ferritin was significantly increased in the pure ACD subgroup as compared to the ACD+IDA subgroup. sTfR and sTfR-F index levels were significantly higher in the ACD+IDA subgroup than in the pure ACD subgroup.

Anemic patients demonstrated significantly higher serum IL-6 and serum hepcidin levels compared to nonanemic patients (Table 2). Patients in the pure ACD subgroup had significantly higher serum IL-6 and serum hepcidin levels compared to patients in the ACD+IDA subgroup.

No significant differences were found between anemic and nonanemic patients regarding lipid profile.

Fifty-three patients with RA (91.6) showed evidence of coronary artery calcification. In the pure ACD subgroup, $75 \%$ of the patients showed moderate evidence of plaque, $12.5 \%$ showed mild evidence of plaque, and $6.25 \%$ showed minimal evidence of plaque. However, in the ACD+IDA subgroup, $20.8 \%$ of the patients showed moderate evidence of plaque, $20.8 \%$ showed mild evidence of plaque, and $45.8 \%$ showed minimal evidence of plaque. In addition, in the nonanemic subgroup, $20 \%$ of the patients showed moderate evidence of plaque, $20 \%$ showed mild evidence of plaque, and $45 \%$ showed minimal evidence of plaque. None of the patients with RA showed extensive coronary plaque by calcium score (Table 3). CCS was significantly higher in the pure ACD subgroup than in the ACD+IDA and nonanemic subgroups. 
Table 2. Clinical, laboratory, and radiographic measures in rheumatoid arthritis (RA) subgroups. Values represent mean $\pm \mathrm{SD}$.

\begin{tabular}{|c|c|c|c|}
\hline Characteristic & $\begin{array}{c}\mathrm{ACD}+\mathrm{IDA} \\
\mathrm{n}=24\end{array}$ & $\begin{array}{l}\text { RA Patients, } n=60 \\
\text { Pure ACD } \\
n=16\end{array}$ & $\begin{array}{l}\text { Nonanemic, } \\
\quad \mathrm{n}=20\end{array}$ \\
\hline Disease duration, yrs & $9.667 \pm 3.655$ & $10.25 \pm 3.13$ & $10.3 \pm 2.90$ \\
\hline DAS28 & $5.36 \pm 0.80 *$ & $5.27 \pm 0.62 *$ & $3.43 \pm 0.64$ \\
\hline MHAQ & $2.25 \pm 0.847^{*}$ & $2.38 \pm 0.72 *$ & $1.5 \pm 0.69$ \\
\hline $\mathrm{ESR}, \mathrm{mm} / \mathrm{h}$ & $45.80 \pm 16.70^{*}$ & $43.75 \pm 9.22 *$ & $27.75 \pm 6.38$ \\
\hline hsCRP, mg/l & $8.27 \pm 4.58^{*}$ & $7.47 \pm 2.64 *$ & $5.675 \pm 2.04$ \\
\hline Rheumatoid factor, IU/ml & $84.29 \pm 39.16$ & $78.31 \pm 23.98$ & $86.9 \pm 33.44$ \\
\hline Total cholesterol, mg/dl & $231.88 \pm 31.89$ & $235.31 \pm 29.92$ & $238.75 \pm 28.33$ \\
\hline Triglyceride, mg/dl & $132.83 \pm 47.81$ & $133.13 \pm 47.96$ & $127.5 \pm 48.73$ \\
\hline LDL-C, mg/dl & $138.5 \pm 26.66$ & $138.63 \pm 25.83$ & $133.9 \pm 24.97$ \\
\hline HDL-C, mg/dl & $42.41 \pm 8.97$ & $45.94 \pm 9.75$ & $43.45 \pm 9.43$ \\
\hline TC/HDL-C & $5.59 \pm 0.99$ & $5.70 \pm 1.08$ & $5.72 \pm 1.12$ \\
\hline LDL/HDL & $3.05 \pm 0.97$ & $3.10 \pm 0.95$ & $2.99 \pm 0.94$ \\
\hline ApoB, g/l & $121.04 \pm 9.97$ & $122.94 \pm 10.44$ & $121.85 \pm 9.87$ \\
\hline ApoA-1 g/1 & $124.83 \pm 18.23$ & $125.63 \pm 17.65$ & $126.55 \pm 16.49$ \\
\hline Hemoglobin, g/dl & $6.06 \pm 1.55^{*, * *}$ & $7.25 \pm 1.85^{*}$ & $13 \pm 0.73$ \\
\hline Serum iron, $\mu \mathrm{g} / \mathrm{dl}$ & $41.5 \pm 20.16^{*}$ & $49.37 \pm 16.9^{*}$ & $84.5 \pm 17.0$ \\
\hline Ferritin, $\mu \mathrm{g} / \mathrm{l}$ & $32.13 \pm 17.87 * \dagger$ & $224.37 \pm 122.20 *$ & $107.5 \pm 5.94$ \\
\hline TIBC, $\mu \mathrm{g} / \mathrm{dl}$ & $415.42 \pm 56.80^{* \dagger}$ & $209.5 \pm 43.09^{*}$ & $230 \pm 48.94$ \\
\hline TfS, $\%$ & $13.12 \pm 11.55^{* \dagger}$ & $19.61 \pm 12.36^{*}$ & $24.86 \pm 22.23$ \\
\hline $\mathrm{sTfR}, \mu \mathrm{g} / \mathrm{ml}$ & $6.22 \pm 2.72 * \dagger$ & $2.36 \pm 0.64$ & $2.16 \pm 0.63$ \\
\hline sTfR-F index & $6.96 \pm 4.87 * \dagger$ & $0.9 \pm 0.85^{*}$ & $1.48 \pm 0.68$ \\
\hline Tumor necrosis factor- $\alpha, \mathrm{pg} / \mathrm{ml}$ & $7.88 \pm 3.01^{*}$ & $7.88 \pm 2.99 *$ & $4.88 \pm 1.50$ \\
\hline Interleukin $6, \mathrm{pg} / \mathrm{ml}$ & $25.65 \pm 12.89^{* \dagger}$ & $50.69 \pm 12.97 *$ & $14.6 \pm 4.90$ \\
\hline Hepcidin, ng/ml & $407.92 \pm 109.70 * \dagger$ & $547.5 \pm 132.89^{*}$ & $351.5 \pm 132.79$ \\
\hline CCS, Agatston units & $123.04 \pm 118.08^{\dagger}$ & $140.94 \pm 129.22 *$ & $119 \pm 118.71$ \\
\hline
\end{tabular}

$* \mathrm{p}<0.001$ vs nonanemic subgroup; $* * \mathrm{p}<0.05$ vs pure ACD (in RA group) ${ }^{\dagger} \mathrm{p}<0.001$ vs pure ACD (in RA group). ACD + IDA: anemia of chronic disease with coexistent iron deficiency anemia; pure ACD: pure anemia of chronic disease; MHAQ: Modified Health Assessment Questionnaire; DAS28: Disease Activity Score for 28 joints; ESR: erythrocyte sedimentation rate; hsCRP: high sensitivity C-reactive protein; LDL-C: low-density lipoprotein cholesterol; HDL-C: high-density lipoprotein cholesterol; ApoB: apolipoprotein B; TIBC: total iron binding capacity; TfS: transferrin saturation; sTfR-F: serum transferrin receptor/log ferritin; CCS: coronary calcium score.

Table 3. Coronary calcium scores in different rheumatoid arthritis (RA) subgroups. Data are number (percentage) of patients. The test of significance is chi-square $=16.15, \mathrm{p}=0.04$.

\begin{tabular}{|c|c|c|c|c|c|}
\hline RA Subgroups & None $(0)$ & Minimal (1-10) & $\begin{array}{c}\text { Coronary Calcium Scores } \\
\text { Mild }(11-100)\end{array}$ & Moderate (101-400) & Extensive (> 400) \\
\hline $\mathrm{ACD}+\mathrm{IDA}, \mathrm{n}=24$ & $3(12.5)$ & $11(45.8)$ & $5(20.8)$ & $5(20.8)$ & $0(0)$ \\
\hline Nonanemic, $\mathrm{n}=20$ & $3(15)$ & $9(45)$ & $4(20)$ & $4(20)$ & $0(0)$ \\
\hline
\end{tabular}

ACD + IDA: anemia of chronic disease with coexistent iron deficiency anemia; pure ACD: pure anemia of chronic disease.

However, there was no significant difference between CCS levels in the ACD+IDA subgroup and the nonanemic subgroup (Table 2).

In patients with RA, serum hepcidin levels were positively correlated with mean disease duration, ESR, hsCRP, RF, DAS28, MHAQ, serum ferritin, IL-6, and mean CCS. However, serum hepcidin levels were inversely correlated with $\mathrm{Hb}$, sTfR levels, and sTfR-F index. No significant correlation was demonstrated between serum hepcidin levels and lipid profile, serum iron, TIBC, TfS percentage, or TNF- $\alpha$ (Table 4).

Multiple linear regression obtained for serum hepcidin levels revealed that there were statistically significant correlated independent factors: hsCRP ( $\beta=0.389 ; \mathrm{p}<0.001)$, RF $(\beta=0.381 ; p<0.001)$, DAS28 $(\beta=0.289 ; p=0.009)$, serum ferritin ( $(=0.292 ; p<0.001)$, IL-6 $(\beta=0.556 ; p<0.001)$, coronary calcium score $(\beta=0.442 ; \mathrm{p}<0.001), \mathrm{Hb}(\beta=-0.321$; $\mathrm{p}<0.001)$, and sTfR-F index $(\beta=-0.355 ; \mathrm{p}<0.001)$. 
Table 4. Correlations between serum hepcidin levels and clinical, laboratory, and radiographic measures in patients with rheumatoid arthritis $(\mathrm{n}=$ 60). Values were calculated using Spearman's rank correlation.

\begin{tabular}{|c|c|c|}
\hline Characteristic & $\mathrm{r}$ & $\mathrm{p}$ \\
\hline Disease duration, yrs & 0.822 & $<0.001$ \\
\hline DAS28 & 0.793 & $<0.001$ \\
\hline MHAQ & 0.693 & $<0.001$ \\
\hline $\mathrm{ESR}, \mathrm{mm} / \mathrm{h}$ & 0.875 & $<0.001$ \\
\hline hsCRP, mg/l & 0.743 & $<0.001$ \\
\hline Rheumatoid factor, IU/ml & 0.863 & $<0.001$ \\
\hline Total cholesterol, mg/dl & 0.222 & NS \\
\hline Triglyceride, mg/dl & -0.085 & NS \\
\hline LDL-C, mg/dl & 0.148 & NS \\
\hline HDL-C, mg/dl & 0.055 & NS \\
\hline TC/HDL-C & 0.134 & NS \\
\hline LDL/HDL & 0.098 & NS \\
\hline ApoB, g/1 & 0.288 & NS \\
\hline ApoA-1, g/1 & 0.075 & NS \\
\hline Hemoglobin, g/dl & -0.842 & $<0.001$ \\
\hline Serum iron, $\mu \mathrm{g} / \mathrm{dl}$ & 0.055 & NS \\
\hline Ferritin, $\mu \mathrm{g} / \mathrm{dl}$ & 0.693 & $<0.001$ \\
\hline $\mathrm{TIBC}, \mu \mathrm{g} / \mathrm{dl}$ & 0.248 & NS \\
\hline TfS, \% & 0.095 & NS \\
\hline $\mathrm{sTfR}, \mu \mathrm{g} / \mathrm{ml}$ & -0.624 & $<0.001$ \\
\hline sTfR-F index & -0.511 & $<0.001$ \\
\hline Tumor necrosis factor- $\alpha, \mathrm{pg} / \mathrm{ml}$ & 0.242 & NS \\
\hline Interleukin $6, \mathrm{pg} / \mathrm{ml}$ & 0.645 & $<0.001$ \\
\hline CCS, Agatston units & 0.672 & $<0.001$ \\
\hline
\end{tabular}

DAS28: Disease Activity Score for 28 joints; ESR: erythrocyte sedimentation rate; hsCRP: high sensitivity C-reactive protein; LDL-C: low-density lipoprotein cholesterol; HDL-C: high-density lipoprotein cholesterol; ApoB: apolipoprotein B; TIBC: total iron binding capacity; TfS: transferrin saturation; sTfR-F: serum transferrin receptor/log ferritin; CCS: coronary calcium score. NS: nonsignificant.

\section{DISCUSSION}

Anemia is a cause of morbidity in patients with RA. ACD is an important phenomenon and iron is a crucial factor of this complex process ${ }^{19}$. In our study, 40 patients $(66.6 \%)$ were anemic, a finding that corresponds with results from Wilson, et $a l^{20}$, who reported a prevalence of anemia of $26.9 \%-77.6 \%$ in patients with RA. Among our anemic patients with RA, 16 (40\%) were found to have pure ACD, while $24(60 \%)$ had ACD+IDA. This agrees with many authors who found that $30 \%$ to $70 \%$ of anemia occurring in patients with RA is attributed to $\mathrm{ACD}^{2,21,22,23}$; however, it disagrees with findings from Goyal, et $a l^{16}$, who found that $20 \%$ of their patients had pure ACD and $80 \%$ had IDA. They attributed this high percentage to the increased prevalence of iron deficiency in the general population of India.

The anemia in RA develops slowly during the first months of illness. There is broad consensus that proinflammatory cytokines play an important role in this syndrome. Accordingly, ACD is also known as "anemia of inflammation" (AI $)^{24}$.

Many studies defined ACD as follows: evidence of chronic inflammation (high CRP level); Hb concentration $<13 \mathrm{~g} / \mathrm{dl}$ for men and $<12 \mathrm{~g} / \mathrm{dl}$ for women; and TfS $<20 \%$, but normal or increased serum ferritin concentration $(>100 \mathrm{ng} / \mathrm{ml})$ or low serum ferritin concentration (30-100 ng/ml), and an sTfR/log ferritin ratio $<1$. These studies also defined ACD+IDA as follows: chronic inflammation (high CRP level); Hb concentration $<13 \mathrm{~g} / \mathrm{dl}$ for men and $<12 \mathrm{~g} / \mathrm{dl}$ for women; and low TfS $<20 \%$, serum ferritin concentration $>30$ and $<100 \mathrm{ng} / \mathrm{ml}$, and an sTfR/log ferritin ratio $>2 .{ }^{25,26}$ Masson $^{27}$ added that serum transferrin levels are normal or high in ACD, TfS is decreased, and sTfR is not increased (a distinguishing feature with IDA). In our study, our anemic patients met the previous proposed definitions for ACD and ACD+IDA.

We observed that anemic patients have a severe active disease as reflected by high disease activity measures (ESR, hsCRP, and DAS28) and MHAQ scores. This observation was also made in other studies ${ }^{23,28}$. Further, it was found that erythropoietin given to treat anemia in patients with RA has led to reduced disease activity, increased muscle strength, and reduced fatigue in some patients ${ }^{4}$.

The pathogenesis of anemia of inflammation was thought to be cytokine-mediated and is due to the typical overexpression of IL-6, which is a major inducer of hepcidin production, leading to hypoferremia ${ }^{29}$. IL-6 has a key role in the systemic inflammatory response, as it is the only cytokine capable of stimulating synthesis of all the acute-phase proteins, including $\mathrm{CRP}^{5}$. When human hepatocytes are exposed to IL-6 but not IL-1 or TNF- $\alpha$, induction of hepcidin messenger RNA may occur $^{30}$. Significantly elevated serum levels of IL-6 and TNF- $\alpha$ have been observed in the sera of our patients. Their levels were significantly higher in sera of anemic compared to nonanemic patients. Nevertheless, only the IL-6 level was significantly higher in the pure ACD subgroup than in the ACD+IDA subgroup. These findings coincide with those from Ganz ${ }^{31}$, who observed large amounts of IL- 6 in the sera and synovial fluids of patients with RA. In addition, serum and synovial IL-6 levels have been found to correlate with RA disease activity 32

In our study, hepcidin concentration followed the same pattern of IL-6 among our patient subgroups and controls, indicating parallel activities. While Dallalio, et $a l^{33}$ stated that serum hepcidin concentrations did not differ significantly between the anemia subsets, many researchers agreed with our findings and reported high levels of hepcidin in patients with RA, levels that were higher in the ACD subgroup than in the ACD+IDA subgroup and in turn, higher than in controls ${ }^{34,35,36}$. The presence of low hepcidin levels in the ACD+IDA subgroup despite detectable CRP levels has been attributed to many factors, such as differences in the half-lives of CRP and hepcidin (the half-life of CRP is $19 \mathrm{~h}$, while the half-life of hepcidin may be much shorter). Another intriguing explanation is the possibility that iron deficiency in these patients may be counterbalancing inflammation-induced hepcidin production. In that situation, low CRP and low hepcidin would indicate iron deficiency and high CRP and high hep- 
cidin would indicate inflammation, while high CRP and low hepcidin would indicate a mixture of inflammation and iron deficiency $^{34}$. These data suggest that hepcidin might play a fundamental role in the pathogenesis of ACD and ACD/IDA, and it could be a potential marker for detection and differentiation of these anemias.

In our study, we postulated that hepcidin levels positively correlated with disease activity, disease severity, serum ferritin, and IL-6, and negatively correlated with $\mathrm{Hb}$ levels. However, no significant correlation was found between serum hepcidin levels and serum iron, TIBC, TfS percentage, or TNF- $\alpha$. Masson ${ }^{27}$ and Demirag, et $a l^{35}$ reported a positive correlation between serum hepcidin and RA activity and a negative correlation with $\mathrm{Hb}$ level. Conversely, Cheng, et al ${ }^{36}$ found no correlation between serum hepcidin and $\mathrm{Hb}$ level. Sasu, et $a^{34}$ documented a strong correlation between serum hepcidin and anemia of inflammation. Dallalio, et $a l^{33}$ found a close association and positive correlation between serum hepcidin and ferritin, but no significant correlation with any other laboratory measures reflecting iron status or degree of anemia. This correlation of hepcidin with disease activity, severity, ferritin, and IL-6 raises the possibility of using hepcidin as a reliable marker of inflammation in RA, and that the interruption of the hepcidin cascade by hepcidin antagonists could be a future treatment for ACD. This was observed by Poli, et $a l^{37}$ when they noted a strong reduction of serum hepcidin in patients treated with heparin to prevent deep vein thrombosis. The treatment was accompanied by an increase of serum iron and a reduction of CRP levels.

In patients with RA, atherosclerotic lesions occur earlier and have a more rapid progression than in the general population and some authors proposed the terms "accelerated atherosclerosis" or "premature atherosclerosis" to underline the magnitude of this process in $\mathrm{RA}^{38}$.

Our study demonstrated increased levels of TC, LDL-C, TG, and apoB with decreased HDL-C and apoA-1 levels in patients with RA. As a consequence, the atherogenic ratio of TC/HDL-C as well as that of LDL-C/HDL-C was significantly higher compared to controls. These changes are associated with an increased incidence of cardiovascular disease in the general population. There have been studies reporting increased, decreased, or similar levels for TC, LDL-C, and HDL-C in comparison to control subjects $39,40,41,42$. The discrepancies in lipid values that were observed in various studies might be due to differences in the populations studied as well as in disease activity.

CCS was significantly higher in our patients with RA than in controls, and higher in the pure ACD subgroup than in the $\mathrm{ACD}+\mathrm{IDA}$ and nonanemic subgroups. However, there was no significant difference between CCS in the ACD+IDA subgroup compared to nonanemic patients. Coronary calcification has long been known to occur as part of the atherosclerotic process; it correlated closely with atherosclerotic plaque formation and thus is a sensitive marker of existing athero- sclerosis $^{43}$. Thus, CCS results in our patients emphasize that inflammation has led to more atherosclerosis in patients with pure ACD. Moreover, the positive correlation found between serum hepcidin levels and mean disease duration, disease activity, serum ferritin, IL-6, and mean CCS clearly demonstrates the interaction between activity and inflammatory measures together with the longer disease duration that collectively have led to anemia of inflammation and consecutively to coronary atherosclerosis.

$\mathrm{Li}$, et $a l^{44}$ suggested that atherosclerosis in ACD occurs because hepcidin causes macrophages within atherosclerotic lesions to be laden with iron. The lesional macrophage iron appears not to be a benign component of the lesion but a crucially important determinant of lesion progression and destabilization. This possibility has been further supported by the fact that iron depletion protects against cardiovascular disease by reducing iron deposition within atherosclerotic plaques and increasing plaque stability ${ }^{45}$.

$\mathrm{Ma}$, et $a l^{46}$ reported that patients with RA who have active disease with longer disease duration have increased risk of cardiovascular events compared to the normal population, a finding that coincides with our previous ones. Meanwhile, Hosseinsabet, et $a l^{47}$ found no correlation between CRP and CCS.

In our patients, traditional cardiovascular risk factors were eliminated during patient selection, except for serum lipids, which were further eliminated on the finding of the lack of its correlation with serum hepcidin. Thus, our results clearly elucidate the role of inflammation per se in coronary atherosclerosis without the interacting role of traditional risk factors. We also clearly show the direct link between hepcidin, anemia of inflammation, and atherosclerosis in our patients.

We conclude that hepcidin can be considered a key inducer of anemia of inflammation in patients with RA. This inflammation was proved to be directly linked to the occurrence of coronary artery atherosclerosis in those patients. The correlations between serum hepcidin with disease activity and IL-6 in our patients have raised the possibility of using hepcidin as a surrogate marker for RA disease activity.

\section{REFERENCES}

1. Wolfe F, Michaud K. Anemia and renal function in patients with rheumatoid arthritis. J Rheumatol 2006;33:1516-22.

2. Fitzsimons EJ, Sturrock RD. The chronic anemia of rheumatoid arthritis: iron banking or blocking? Lancet 2002;360:1713-4.

3. Bowman SJ. Hematological manifestations of rheumatoid arthritis. Scand J Rheumatol 2002;31:251-9.

4. Raj DS. Role of interleukin-6 in the anemia of chronic disease. Semin Arthritis Rheum 2009;38:382-8.

5. Moreland LW, Curtis JR. Systemic nonarticular manifestations of rheumatoid arthritis: focus on inflammatory mechanisms. Semin Arthritis Rheum 2008;39:132-43.

6. Lou DQ, Lesbordes JC, Nicolas G, Viatte L, Bennoun M, Van Rooijen N, et al. Iron- and inflammation-induced hepcidin gene expression in mice is not mediated by Kupffer cells in vivo. Hepatology 2005;41:1056-64.

7. Means RT Jr. Hepcidin and anemia. Blood Reviews

Personal non-commercial use only. The Journal of Rheumatology Copyright @ 2011 . All rights reserved. 
2004;18:219-25.

8. Dessein PH, Joffe BI, Singh S. Biomarkers of endothelial dysfunction, cardiovascular risk factors and atherosclerosis in rheumatoid arthritis. Arthritis Res Ther 2005;7:R634-43.

9. Budoff MJ, Gul KM. Expert review on coronary calcium. Vasc Health Risk Manag 2008;4:315-24.

10. Aletaha D, Neogi T, Silman AJ, Funovits J, Felson DT, Bingham $\mathrm{CO}$, et al. Rheumatoid arthritis classification criteria: an American College of Rheumatology/European League Against Rheumatism collaborative initiative. Ann Rheum Dis 2010;69:1580-8.

11. Prevoo ML, van't Hof MA, Kuper HH, van Leeuwen MA, van de Putte LP, van Riel PL. Modified disease activity scores that include twenty-eight-joint counts. Development and validation in a prospective longitudinal study of patients with rheumatoid arthritis. Arthritis Rheum 1995;38:44-8.

12. Pincus T, Summey JA, Soraci SA Jr, Wallston KA, Hummon NP. Assessment of patient satisfaction in activities of daily living using a modified Stanford Health Assessment Questionnaire. Arthritis Rheum 1983;26:1346-53.

13. Franchini M, Montagnana M, Lippi G. Hepcidin and iron metabolism: From laboratory to clinical implications. Clin Chim Acta 2010;11:21-2.

14. Schwarz P, Strnad P, von Figura G, Janetzko A, Krayenbühl P, Adler G, et al. A novel monoclonal antibody immunoassay for the detection of human serum hepcidin. J Gastroenterol 2011;46:648-56.

15. Giannouli S, Voulgarelis M, Ziakas PD, Tzioufas AG. Anaemia in systemic lupus erythematosus: from pathophysiology to clinical assessment. Ann Rheum Dis 2006;65:144-8.

16. Goyal R, Das R, Bambery P, Garewal G. Serum transferrin receptor-ferritin index shows concomitant iron deficiency anemia and anemia of chronic disease is common in patients with rheumatoid arthritis in north India. Indian J Pathol Microbiol 2008;51:102-4.

17. Agatston AS, Janowitz WR, Hildner FJ, Zusmer NR, Vaimonte M Jr, Detrano R. Quantification of coronary artery calcium using ultrafast computed tomography. J Am Coll Cardiol 1990;15:827-32.

18. Shaw LJ, Raggi P, Schisterman E, Berman DS, Callister TQ. Prognostic value of cardiac risk factors and coronary artery calcium screening for all-cause mortality. Radiology 2003;228:826-33.

19. Formanowicz D, Sackmann A, Kozak A, Blazewicz J, Formanowicz P. Some aspects of the anemia of chronic disorders modeled and analyzed by petri net based approach. Bioprocess Biosyst Eng 2011;34:581-95.

20. Wilson A, Yu HT, Goodnough LT, Nissenson AR. Prevalence and outcomes of anemia in rheumatoid arthritis: a systematic review of the literature. Am J Med 2004;116 Suppl 7A:50S-7S.

21. Baer AN, Dessypris EN, Krantz SB. The pathogenesis of anemia in rheumatoid arthritis: a clinical and laboratory analysis. Semin Arthritis Rheum 1990;19:209-23.

22. Doube A, Davis M, Smith JG, Maddison PJ, Collins AJ. Structured approach to the investigation of anemia in patients with rheumatoid arthritis. Ann Rheum Dis 1992;51:469-72.

23. Agrawal S, Misra R, Aggarwal A. Anemia in rheumatoid arthritis: high prevalence of iron-deficiency anemia in Indian patients. Rheumatol Int 2006;26:1091-5.

24. Weiss G, Goodnough LT. Anemia of chronic disease. N Engl J Med 2005;352:1011-23.

25. Nemeth E. Iron regulation and erythropoiesis. Curr Opin Hematol 2008;15:169-75.

26. Pinto JP, Ribeiro S, Pontes H, Thowfeequ S, Tosh D, Carvalho F, et al. Erythropoietin mediates hepcidin expression in hepatocytes through EPOR signaling and regulation of $\mathrm{C} / \mathrm{EBP}$ alpha. Blood 2008;111:5727-33.
27. Masson C. Rheumatoid anemia. Joint Bone Spine 2011;78:131-7.

28. Borah DJ, Iqbal F. Anemia in recent onset rheumatoid arthritis. JK Science 2007;9:120-2.

29. Song SN, Tomosugi N, Kawabata H, Ishikawa T, Nishikawa T, Yoshizaki K. Down-regulation of hepcidin resulting from long-term treatment with an anti-IL-6 receptor antibody (tocilizumab) improves anemia of inflammation in multicentric Castleman disease. Blood 2010;116:3627-34.

30. Nemeth E, Valore EV, Territo M, Schiller G, Lichtenstein A, Ganz T. Hepcidin, a putative mediator of anemia of inflammation, is a type II acute-phase protein. Blood 2003;101:2461-3.

31. Ganz T. Hepcidin - a regulator of intestinal iron absorption and iron recycling by macrophages. Best Pract Res Clin Haematol 2005; 18:171-82.

32. Fonseca JF, Santos MJ, Canhão H, Choy E. Interleukin-6 as a key player in systemic inflammation and joint destruction. Autoimmun Rev 2009;8:538-42.

33. Dallalio G, Fleury T, Means RT. Serum hepcidin in clinical specimens. Br J Haematol 2003;122:996-1000.

34. Sasu BJ, Li H, Rose MJ, Arvedson TL, Doellgast G, Molineux G. Serum hepcidin but not prohepcidin may be an effective marker for anemia of inflammation (AI). Blood Cells Mol Dis 2010;45:238-45.

35. Demirag MD, Haznedaroglu S, Sancak B, Konca C, Gulbahar O, Ozturk MA, et al. Circulating hepcidin in the crossroads of anemia and inflammation associated with rheumatoid arthritis. Intern Med 2009;48:421-6.

36. Cheng PP, Jiao XY, Wang XH, Lin JH, Cai YM. Hepcidin expression in anemia of chronic disease and concomitant iron-deficiency anemia. Clin Exp Med 2011;11:33-42.

37. Poli M, Girelli D, Campostrini N, Maccarinelli F, Finazzi D, Luscieti S, et al. Heparin: a potent inhibitor of hepcidin expression in vitro and in vivo. Blood 2011;117:997-1004.

38. Tanasescu C, Jurcut C, Jurcut R, Ginghina C. Vascular disease in rheumatoid arthritis: From subclinical lesions to cardiovascular risk. Eur J Intern Med 2009;20:348-54.

39. Lakatos J, Harsagyi A. Serum total, HDL, LDL cholesterol, and triglyceride levels in patients with rheumatoid arthritis. Clin Biochem 1988;21:93-6.

40. Kavanaugh A. Dyslipoproteinaemia in a subset of patients with rheumatoid arthritis. Ann Rheum Dis 1994;53:551-2.

41. Asanuma Y, Kawai, S, Aoshima H, Kaburaki J, Mizushima Y. Serum lipoprotein(a) and apolipoprotein(a) phenotypes in patients with rheumatoid arthritis. Arthritis Rheum 1999;42:443-7.

42. Georgiadis AN, Papavasiliou EC, Lourida ES, Alamanos Y, Kostara $\mathrm{C}$, Tselepis AD, et al. Atherogenic lipid profile is a feature characteristic of patients with early rheumatoid arthritis: effect of early treatment - a prospective, controlled study. Arthritis Res Ther 2006;8:R82.

43. Raggi $\mathrm{P}$, Achenbach S. Computed tomography for atherosclerosis and coronary artery disease imaging. Discov Med 2010;9:98-104.

44. Li W, Ostblom M, Xu LH, Hellsten A, Leanderson P, Liedberg B, et al. Cytocidal effects of atheromatous plaque components: the death zone revisited. FASEB J 2006;20:2281-90.

45. Sullivan JL. Iron in arterial plaque: modifiable risk factor for atherosclerosis. Biochim Biophys Acta 2009;1790:718-23.

46. Ma NH, Teh CL, Rapaee A, Lau KB, Fong AY, Hi S, et al. Subclinical coronary artery disease in Asian rheumatoid arthritis patients who were in remission: a pilot study. Int J Rheum Dis 2010;13:223-9.

47. Hosseinsabet A, Mohebbi A, Almasi A. Association between $\mathrm{C}$-reactive protein and coronary calcium score in coronary artery disease. Cardiovasc J Afr 2009;20:107-11. 